\title{
Evaluation of spatial distribution and characterization of wall shear stress in carotid sinus based on two-dimensional color Doppler imaging
}

\author{
Bo Zhang ${ }^{*+} \mathbb{0}$, Yuqin $\mathrm{Ma}^{\dagger}$ and Fang Ding
}

\author{
${ }^{*}$ Correspondence: \\ zhangbodongfang@qq.com \\ ${ }^{\dagger}$ Bo Zhang and Yuqin $\mathrm{Ma}$ \\ contributed equally to this \\ paper \\ Department of Ultrasound \\ in Medicine, Shanghai East \\ Hospital, Tongji University \\ School of Medicine, \\ Shanghai 200120, China
}

\begin{abstract}
Objective: This study aims to use a wall shear stress (WSS) quantitative analysis software to analyze and evaluate the carotid sinus WSS spatial distribution and characteristics in intima-media thickness (IMT) normal and thickening group by using two-dimensional color doppler flow imaging (CDFI) so as to assist clinicians to predict the location and risk of plaque formation.
\end{abstract}

Methods: According to IMT, 50 subjects was selected as IMT thickening group and 50 subjects as IMT normal group from subjects who had a carotid ultrasound examination in Shanghai East hospital during October 2016 to October 2017. This study presents the spatial distribution of the carotid sinus WSS based on the WSS quantitative analysis software and compared the spatial distribution and characteristics of the carotid sinus WSS between IMT thickening group and IMT normal group through two- and threedimensional WSS maps and a fused WSS image.

Results: The distributional regularity of WSS in both two group was: carotid sinus $<$ common carotid artery (CCA) < internal carotid artery (ICA) and posterior-interior wall of the carotid sinus $<$ the anterior-lateral wall of the carotid sinus. Furthermore, the WSS of CCA, ICA, the anterior-lateral proximal wall of the carotid sinus, the anteriorlateral distal wall of the carotid sinus, the posterior-interior proximal wall of the carotid sinus, and the posterior-interior distal wall of the carotid sinus in IMT thickening group was lower than the corresponding part of IMT normal group $(P<0.05)$.

Conclusion: In summary, this WSS quantitative analysis framework by two-dimensional CDFI can measure and reflect the carotid sinus WSS spatial distribution and characteristics more accurately and visually. As a convenient tool, it may be used for clinical prediction of the plaque formation in carotid sinus in the future.

Keywords: Wall shear stress, Intima-media thickness, Atherosclerosis, Carotid sinus 


\section{Background}

Cardio-cerebral vascular disease is a common disease with high morbidity and mortality in China, which has gradually become is a major public health concern [1]. Cardio-cerebral vascular disease is a general term for cardiovascular disease and cerebrovascular disease [2]. Previous studies have shown that atherosclerosis and early plaque rupture are the main risk factors for myocardial infarction and cerebral ischemic stroke [3]. Intima-media is the earliest site of the occurrence and development of atherosclerosis [4]. The endothelial disfunction, foam cells accumulated in the subintima, subintimal fatty deposited becoming fatty streaks, all above result in intima-media thickening [5]. With the development of atherosclerosis, the intima-media becomes thickening into plaque formation, which is a typical sign of atherosclerosis [6]. Luminal stenosis is the late stage of atherosclerosis and sometimes need endovascular revascularization $[7,8]$. Therefore, it is generally considered that intima-media thickening is an important manifestation of atherosclerosis at early stage. Intima-media thickening and plaque formation are most likely to occur in the carotid sinus [9]. Because of no specific clinical symptoms, intima-media thickness (IMT) thickening or plaque formation have a deficiency for early diagnosis and prediction of atherosclerotic related diseases [10]. In the past few decades, there has been increasing evidence showed that low level, large gradient and high oscillatory wall shear stress (WSS) can destroy the inner intima and endothelial cells and accelerate the intima-media thickening and carotid atherosclerosis $[11,12]$. Furthermore, the rupture of vulnerable plaques is usually observed on the shoulder of the plaque, where it is considered to be the highest of WSS [13]. Therefore, the evaluation of hemodynamic parameters such as changes in local WSS is of great importance for predicting the location of plaque rupture. Among the existing medical imaging modalities, ultrasound, as a non-invasive imaging, is the first choice for analysis of human anatomical structure, which is widely applied to clinical study for better structural visualization when compared with magnetic resonance angiography (MRA), and computed tomography angiography (CTA) [14]. Previously, the Hagen-Poiseuille equation was the most common method to evaluate WSS reasonably and accurately [15]. But this technique could not evaluate local WSS, especially WSS near irregular intravascular plaques [16]. Therefore, Hagen-Poiseuille equation is not feasible as a clinical method to evaluate local WSS around plaque by obtaining stable vessel diameter and velocity [17]. With the development of modern ultrasound technology, such as color Doppler flow imaging (CDFI) and contrast-enhanced ultrasound, not only can average WSS be measured, but also arterial hemodynamic distribution can be measured and visualized in clinical examination [18]. This may lead to modern patterns of diagnosis for plaque and atherosclerosis. In this study, through two-dimensional CDFI, the spatial distribution and characteristics of local WSS in carotid sinus were analyzed and discussed by our proposed WSS quantitative analysis software.

\section{Methods}

\section{Study participants and grouping}

According to IMT, 50 subjects (IMT $\leq 0.9 \mathrm{~mm}$ ) was selected as IMT thickening group and 50 subjects $(0.9 \mathrm{~mm}<\mathrm{IMT} \leq 1.2 \mathrm{~mm})$ as IMT normal group from subjects who had a carotid ultrasound examination by an experienced sonographer (Fang Ding) in Shanghai 
East hospital during October 2016 to October 2017. Exclusion criteria: severe arrhythmia, heart failure and aortic stenosis. The Ethics Committee of the Shanghai East Hospital approved the study, and written informed consent was obtained from all subjects.

\section{Inspection methods}

The Philips IE33 system (Philips Medical Systems, Andover, MA, USA) with a L11-3 linear array transducer was used in our study. Before the carotid ultrasound examination, all subjects took a 10-min rest and no taking irritating food such as coffee and tea. The subjects took the supine position with the neck exposed and the head shifted to the opposite side. We firstly adjusted the speed range to fill the lumen with full blood flow without aliasing, and then selected a proper sampling frame range in order to keep the Doppler graphics frame frequency between 20 and 30 frames.

\section{Conventional parameters of carotid ultrasound}

The conventional data of carotid ultrasound was collected, such as the internal diameter of the CCA, IMT, the peak systolic velocity (PSV) and the vascular resistance index (RI). Then, four locations of carotid sinus in both two group were measured, namely the anterior-lateral proximal wall, the anterior-lateral distal wall, the posterior-interior proximal and the posterior-interior distal wall. The conventional WSS was estimated by traditional Hagen-Poiseuille formula: $\tau_{w}=\frac{2 \mu u_{m}}{R}$ [17]. Here, $\tau_{w}$ is WSS, $\mu$ is blood viscosity, $u_{m}$ is the highest velocity of flow (velocity at the center of the lumen), and $R$ is the inner radius of the lumen.

\section{WSS quantitative analysis by CDFI}

Three long axis dynamic images of the CCA in 3 cardiac cycles were obtained, including the distal end of CCA (about $2 \mathrm{~cm}$ below the CCA bifurcation), the carotid sinus (between the end of the CCA bifurcation and the beginning of the ICA), the proximal end of the ICA. All CDFI images were saved in DICOM (image matrix, $600 \times 800$; pixel spacing, $0.085 \times 0.085 \mathrm{~mm}$ ) format and imported offline to the WSS quantitative analysis software by the MATLAB (The Mathworks Inc. Natick, MA, USA) platform to analyze. Then, the WSS of each pixel in CDFI, the two- and three-dimensional spatial distribution WSS maps and a fused WSS image were obtained as shown in Fig. 1. In this figure, A1 and B1 pertains to the CDFI of Carotid Sinus and the fusion image of WSS distribution with ultrasonic image. A2 and B2 were the segmented CDFI of Carotid Sinus and the two-dimensional distribution of WSS. A3 and B3 were the three-dimensional distribution of blood velocity profile in Carotid Sinus and the three-dimensional distribution of WSS. WSS calculated equation was $\tau_{w}=\mu \frac{d u}{d r} \mid r=$ wall, with the assumption that blood is a Newtonian fluid. In this equation, $\tau_{w}$ is WSS, which is the shear stress near the vessel wall. $\mu$ is blood viscosity. $\frac{d u}{d r}$ is velocity gradient (or shear rate). $r=$ wall means that $r$ is near the boundary of tube. Detailed WSS quantitative analysis steps were reported in our previous study [18]. Based on obtaining the spatial distribution of the two-dimensional WSS imaging, we then collected six regional locations, namely posterior wall of CCA, posterior wall of ICA, posterior-interior wall of the proximal carotid sinus, posterior-interior wall of the distal carotid sinus, the anterior-lateral wall of the proximal carotid sinus, as well as the anterior-lateral wall of the distal carotid sinus. 


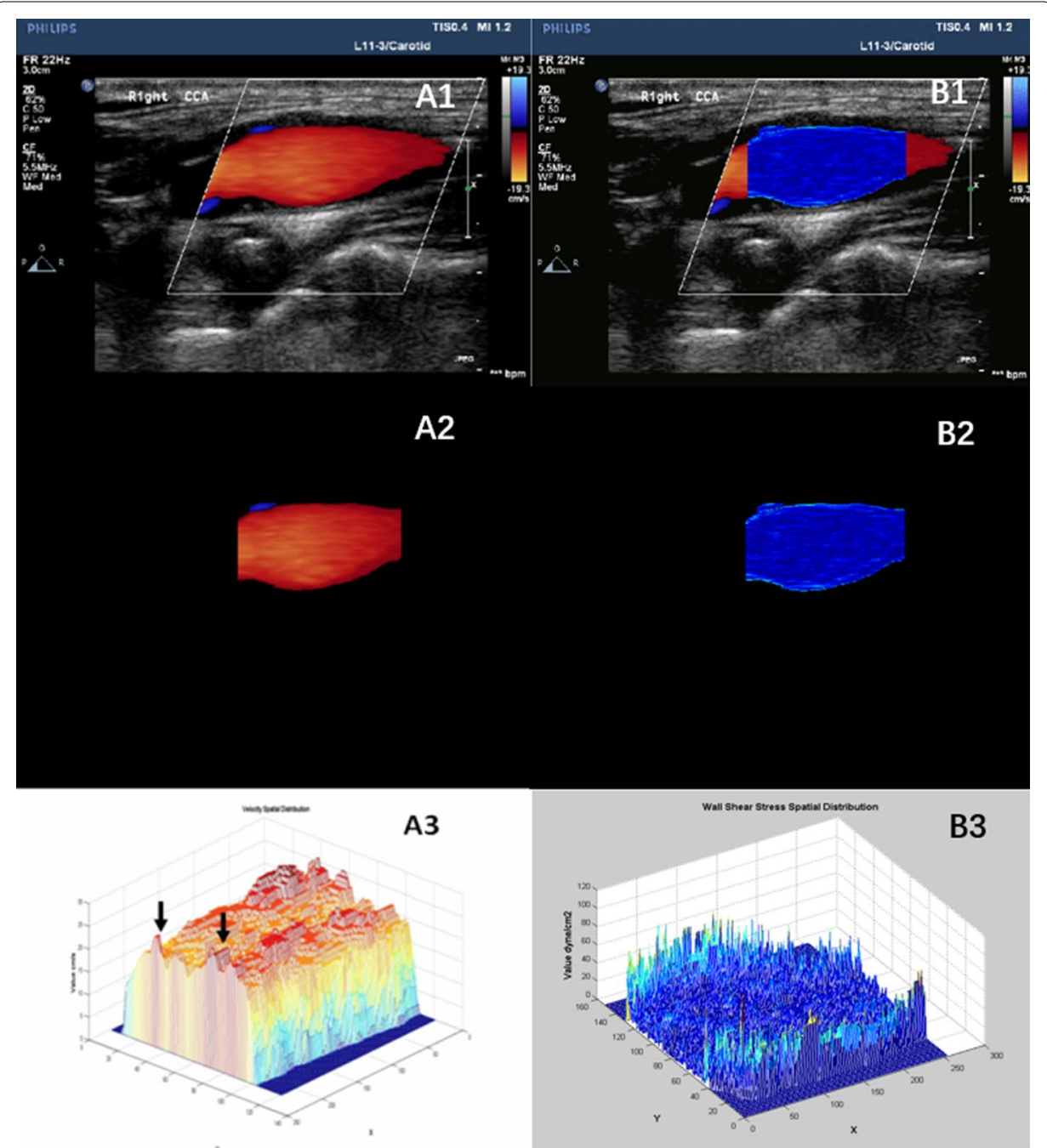

Fig. 1 WSS spatial distribution of normal carotid sinus by WSS quantitative analysis software

The sampling point is shown in Fig. 2. The average WSS of carotid sinus was computed based on the above four values. After recording, the software can automatically import the Excel form in the background and calculate the WSS distribution range and average value of each area of interest.

\section{Statistical analysis}

All WSS data were analyzed by Microsoft Excel and SPSS 22.0 software (SPSS Inc., Chicago, IL, USA). Shapiro-Wilk test was used to assess the distribution of continuous variables. Results were expressed as frequencies and percentages for categorical variables, mean \pm standard deviation (SD) for normally distributed variables and median (interquartile range) for non-normally distributed variables. Comparisons between continuous variables were performed using Independent-Samples T-test, Mann-Whitney U-test, One Way ANOVA test or Kruskal-Wallis test as appropriate. Chi square test was used for comparison of categorial variables. Correlations between the IMT and local 


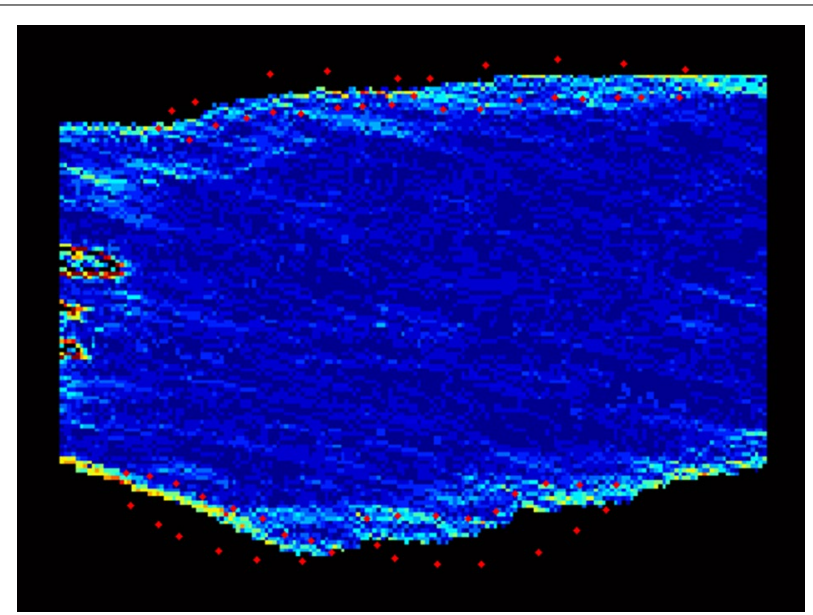

Fig. 2 Two-dimensional spatial distribution of WSS sampling point in the carotid sinus

WSS of carotid sinus was verified by Pearson correlation coefficients. $\mathrm{P}<0.05$ is considered significant for all described analyses.

\section{Result}

\section{Clinical parameters}

The general clinical indexes of the IMT normal group and the IMT thickening group are summarized in Table 1. There was no significant difference in age, sex, height, weight and heart rate between the two groups $(\mathrm{P}>0.05)$. However, the cases of hypertension, coronary heart disease, diabetes, hyperlipidemia and smoking were statistically different $(\mathrm{P}<0.05)$

\section{Conventional parameters of carotid ultrasound}

The conventional carotid ultrasound parameters of the two groups were compared in Table 2. There was no significant difference in the internal diameter of CCA, PSV and RI

Table 1 Clinical characteristics in the IMT normal group and the IMT thickening group

\begin{tabular}{llll}
\hline Variable & $\begin{array}{l}\text { The IMT normal group, } \\
\mathbf{n = 1 0 0}\end{array}$ & $\begin{array}{l}\text { The IMT thickening group, } \\
\mathbf{n = 5 0}\end{array}$ & P value \\
\hline Age (years) & $55.68 \pm 13.46$ & $69.12 \pm 11.62$ & 0.951 \\
Sex (male/female) & $44 / 56$ & $28 / 22$ & 0.166 \\
Height (cm) & $170.47 \pm 6.32$ & $173.25 \pm 7.11$ & 0.084 \\
Weight (kg) & $65.32 \pm 15.87$ & $70.39 \pm 16.45$ & 0.072 \\
Heart rate (bpm) & $73.25 \pm 7.14$ & $68.58 \pm 6.22$ & 0.545 \\
Hypertension (yes/no) & $32 / 68$ & $42 / 8$ & $0.000^{*}$ \\
CHD (yes/no) & $14 / 86$ & $32 / 28$ & $0.000^{*}$ \\
Diabetes (yes/no) & $4 / 96$ & $12 / 38$ & $0.000^{*}$ \\
Hyperlipidemia (yes/no) & $5 / 95$ & $18 / 32$ & $0.000^{*}$ \\
Smoking (yes/no) & $22 / 78$ & $24 / 26$ & $0.001^{*}$ \\
\hline
\end{tabular}

CHD coronary heart disease

${ }^{*} \mathrm{P}<0.05$ 
Table 2 Conventional carotid ultrasound parameters in the IMT normal group and the IMT thickening group

\begin{tabular}{llll}
\hline Variable & IMT normal group, $\mathbf{n = 1 0 0}$ & $\begin{array}{l}\text { IMT thickening group, } \\
\mathbf{n = 5 0}\end{array}$ & P value \\
\hline CCA inner diameter $(\mathrm{mm})$ & $6.91 \pm 0.5$ & $6.73 \pm 0.4$ & 0.257 \\
CCA IMT (mm) & $0.69 \pm 0.16$ & $0.99 \pm 0.19$ & $0.000^{*}$ \\
PSV (cm/s) & $91.3 \pm 20.7$ & $95.4 \pm 18.8$ & 0.188 \\
RI & $0.72 \pm 0.05$ & $0.69 \pm 0.08$ & 0.358 \\
WSS of CCA & $7.81 \pm 2.72$ & $6.94 \pm 1.38$ & $0.034^{*}$ \\
\hline
\end{tabular}

* $\mathrm{P}<0.05$

a Computed by Hagen-Poiseuille formula

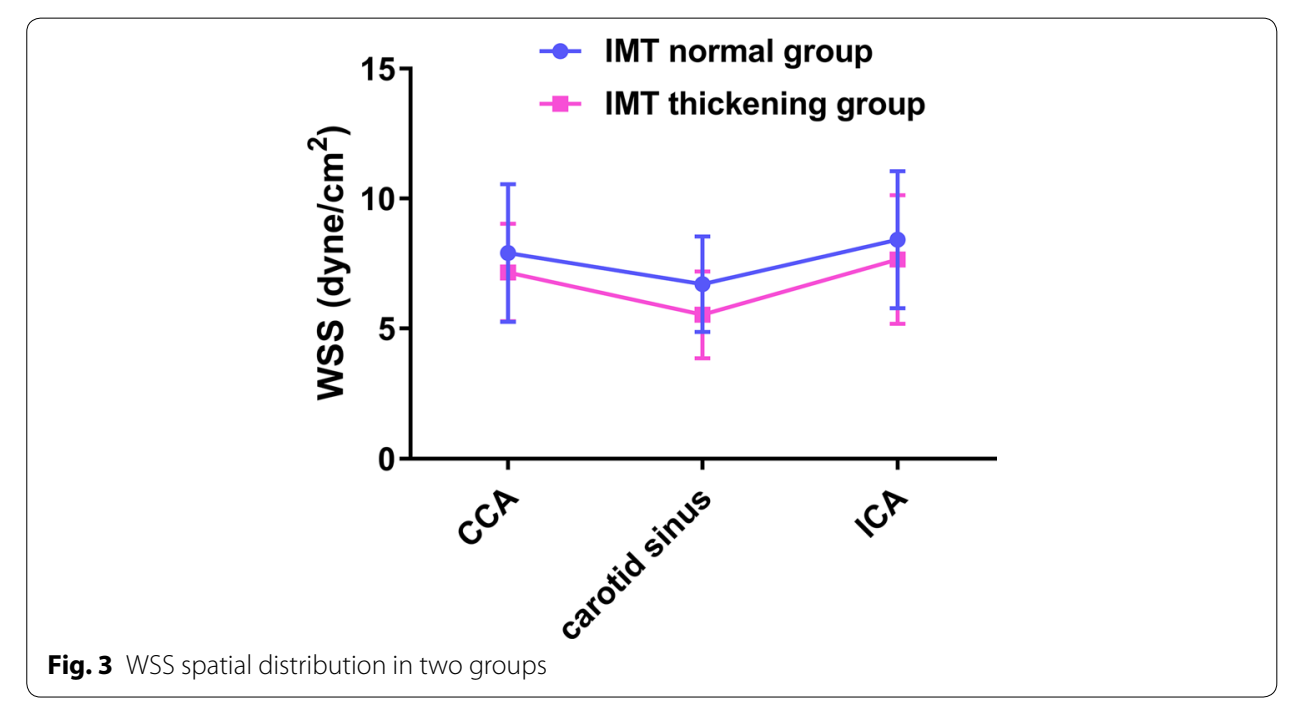

between the two groups. The conventional WSS of CCA in IMT thickening group was lower than that in normal group.

\section{WSS quantitative analysis}

\section{Respective WSS spatial distribution in two group}

As shown in Fig. 3, we can preliminarily find the WSS of carotid sinus $<$ CCA $<$ ICA in both IMT normal group and IMT thickening group.

As shown in Figs. 4 and 5, there was no significant difference between the proximal and distal ends of the carotid sinus $(\mathrm{P}>0.05)$, while the anterior-lateral wall of carotid sinus $<$ posterior-interior wall in both two group $(\mathrm{P}<0.05)$.

\section{Spatial distribution of WSS between IMT thickening group and normal group}

Comparing the two groups (Table 3), we found that the WSS of CCA, ICA, proximal and distal ends of the anterolateral wall, proximal and distal ends of the posterolateral wall of the carotid sinus in IMT thickening group were lower than those of IMT normal group $(\mathrm{P}<0.05)$. 
Table 3 WSS in the IMT normal group and the IMT thickening group

\begin{tabular}{lccc}
\hline WSS $\left(\mathbf{d y n e} / \mathbf{c m}^{\mathbf{2}}\right.$ ) & IMT normal group, $\mathbf{n = 1 0 0}$ & IMT thickening group, $\mathbf{n = 5 0}$ & P value \\
\hline CCA & $7.91 \pm 2.65$ & $7.15 \pm 1.88$ & $0.027^{*}$ \\
ICA & $8.42 \pm 2.64$ & $7.65 \pm 2.48$ & $0.031^{*}$ \\
ALPW of carotid sinus & $6.02 \pm 1.79$ & $5.08 \pm 1.06$ & $0.025^{*}$ \\
ALDW of carotid sinus & $5.98 \pm 2.01$ & $5.33 \pm 1.56$ & $0.001^{*}$ \\
PIPW of carotid sinus & $7.52 \pm 2.36$ & $5.86 \pm 1.76$ & $0.001^{*}$ \\
PIDW of carotid sinus & $7.31 \pm 1.25$ & $5.85 \pm 2.04$ & $0.034^{*}$
\end{tabular}

$A L P W$ the anterior-lateral proximal wall, $A L D W$ the anterior-lateral distal wall, $P I P W$ the posterior-interior proximal wall, $P I D W$ the posterior-interior distal wall

* $\mathrm{P}<0.05$

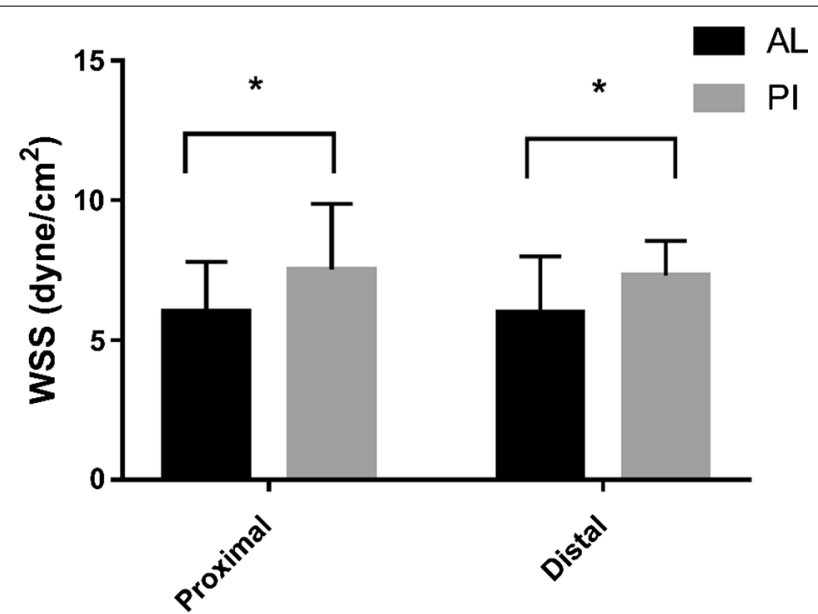

Fig. 4 WSS of carotid sinus in IMT normal group. * $P<0.05$; $A L$ the anterior-lateral, $P$ I the posterior-interior

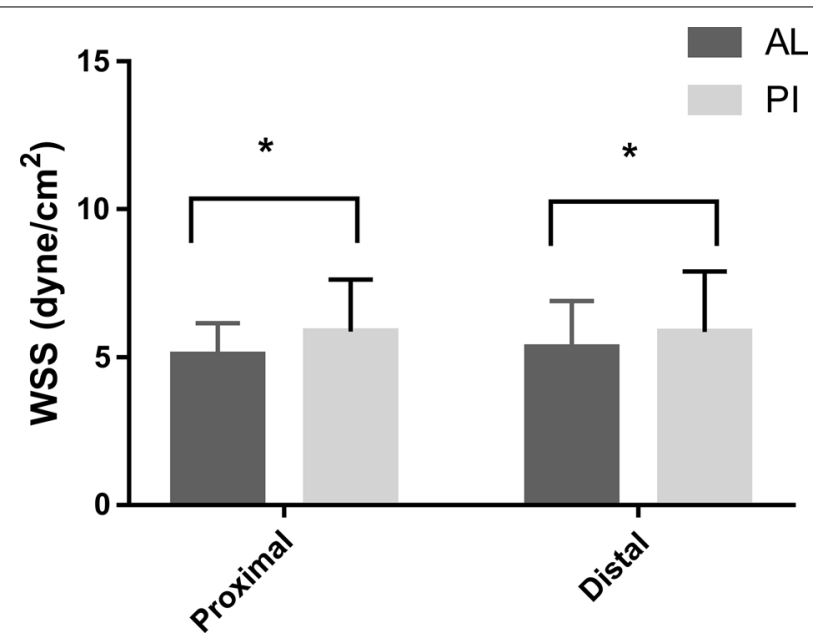

Fig. 5 WSS of carotid sinus in IMT thickening group. ${ }^{*} P<0.05$; AL the anterior-lateral, $P$ I the posterior-interior

The correlation between the IMT and local WSS of carotid sinus

On account of 4 regional locations in each carotid sinus, there were total 200 locations in IMT thickening group, among which 122 IMT thickening locations and 78 IMT 
Table 4 WSS in IMT thickening regions and IMT normal regions of carotid sinus

\begin{tabular}{lccc}
\hline Carotid sinus & Numbers & WSS & P value \\
\hline IMT thickening location & 122 & $4.18 \pm 1.35$ & $0.035^{*}$ \\
IMT normal location & 78 & $7.93 \pm 2.07$ & \\
\hline${ }^{*} \mathrm{P}<0.05$ & & &
\end{tabular}

* $P<0.05$

normal locations. Furthermore, the WSS of the former locations was smaller than the latter $(\mathrm{P}<0.05)$, as shown in Table 4 . Then a bivariate correlation analysis between the IMT of 200 locations corresponding to WSS results in correlated coefficient $r=-0.352$ $(\mathrm{P}=0.000)$, showing a negative weak correlation.

\section{Discussion}

\section{Traditional risk factors for atherosclerosis}

Atherosclerosis remains the leading cause of death and morbidity worldwide. Noninvasive imaging techniques such as ultrasound are currently available for the detection of subclinical atherosclerosis and assessment of its progression and regression [19]. Previous studies had proved that hypertension, dyslipidemia, diabetes, obesity and smoking are some traditional risk factors for cardio-cerebrovascular diseases [20]. By comparison with the clinical data between the IMT thickening group and the normal group, we found that the incidence of hypertension, coronary heart disease, diabetes, hyperlipidemia and smoking in the IMT thickening group are higher than the IMT normal group, which also proved that these diseases are closely related to atherosclerosis. However, such traditional risk factors only reflected the susceptibility of atherosclerosis and could not demonstrate the spatial distribution and dynamic development of atherosclerosis in individuals. Therefore, we need to find some other sensitive and accurate index to predict the potential development of atherosclerosis and plaque formation at early stage.

\section{Evaluation indexes and methods of atherosclerosis}

The most common clinical detection of atherosclerosis is to measure IMT by using ultrasonic imaging. The internal diameter of CCA, IMT, PSV and RI were recorded by conventional vascular ultrasound, and the conventional WSS was estimated by traditional formula Hagen-Poiseuille, $\tau_{w}=\frac{2 \mu u_{m}}{R}$. There was no significant difference in internal diameter of CCA, PSV and RI between the two groups $(\mathrm{P}>0.05)$, but the WSS of CCA $\tau_{w}$ in IMT thickening group was significantly lower $(\mathrm{P}<0.05)$. We can conclude that the conventional parameters from carotid ultrasound are not sensitive in detecting atherosclerosis. The possible reason is that atherosclerosis is a long-term process from quantitative accumulation to qualitative transformation $[5,6]$. When the vascular wall at the early stage of IMT thickening, there is no obvious changes in vascular morphology or regional hemodynamics [21]. In contrast to these conventional evaluation indexes, the WSS calculated by formula Hagen-Poiseuille showed a difference between the IMT thickening group and the IMT normal group. This suggests that WSS may be a sensitive indication for early evaluation of atherosclerosis, which is consistent with previous studies on the relationship between WSS and atherosclerosis mentioned in the background [22]. According to the principle of Hagen-Poiseuille formula, the equation is based on 
the diameter of the vessel and the maximum flow velocity and represents the value of WSS of all points in the cross section of the vascular wall [23]. However, the arterial vessel of human is not likely to be standard round, and the WSS will be affected by different factors such as blood flow, blood pressure, geometric structure of the vessel wall, IMT, etc., which will result in different WSS values at each point of the vessel wall [24]. Therefore, the results calculated by the Hagen-Poiseuille formula can only be used as rough estimates, which cannot reflect the local WSS changes near the vascular wall. In addition, the Hagen-Poiseuille formula were mainly used to estimate large arteries with cylindrical tube and rigid wall such as CCA. It is impossible to accurately evaluate the WSS of irregular blood vessel such as carotid sinus with a local intumescentia [25]. The WSS quantitative analysis framework that is used in this study is developed independently and based on MATLAB platform. Our software makes use of digital image processing technology to extract the blood flow signal from CDFI. The color data of each pixel in CDFI is converted into blood flow velocity data and then converted into shear stress data according to the definition formula of WSS. Theoretically, the WSS distribution of blood vessels with any shape can be analyzed. In previous study, the design principle of the WSS quantitative analysis software was expounded and it was used to measure the spatial distribution of WSS in the normal CCA in vivo [18]. Zhang et al. compared the accuracy of WSS to Hagen-Poiseuille formula, which shows that this method can hopefully present more accurate and detailed values of regional WSS near vessel walls [26].

\section{Hemodynamic changes at carotid sinus}

Carotid sinus is one special bifurcation of carotid artery with complex structure and individual geometry, where hemodynamics changes suddenly and the blood flow is turbulent [27]. On account of the blood flow shunting to ICA and ECA, the WSS of carotid sinus altered. Previous studies have found that the inner wall of two sub-vessels in the of the carotid bifurcation divides the blood flow into two tributaries, and the corresponding wall is subjected to the largest axial impact forming a high shear stress region [28]. Owning to the blood flow of the carotid bifurcation flowing into the sub-vessels at a certain angle, so the lateral wall of branch cavity inlet is turbulent due to the flow separation and then forms a low WSS region [29]. But in the past studies, most WSS data were acquired from artificial models, so the observed data in vivo was not enough. This study is based on CDFI to evaluate the distribution of WSS and changing regularities in carotid sinus in vivo. Hemodynamic changes occurring at the initial segments of the arterial bifurcations play a vital role in the development of atherosclerotic plaque, so atherosclerotic plaque is likely to locate in carotid sinus [30]. So under such condition of noninvasive methodology, the self-developed WSS quantitative analysis software combined with texture matching method can detect the early diagnosis of atherosclerosis and can obtain specific WSS for further evaluation.

\section{Limitations and future developments}

Firstly, the WSS data from CDFI in our study are not three-dimensional data as compared to MRA. However, ultrasound scanning is simple, time-saving and relatively economical compared with MRA, which makes WSS assessment easier to apply. In 
addition, our CDFI-based program cannot be applied for detecting the WSS of the coronary artery, which can be further assessed by angiography or intravascular ultrasound. At last, the outcome in IMT thickening group was not been long-term followed. Therefore, a large long-term followed study subject group is required to confirm the development of atherosclerosis.

\section{Conclusion}

In summary, WSS of IMT thickening location is becoming lower in early stage of atherosclerosis. This variation was showed in CCA, ICA, and all parts of the carotid sinus. The distribution of WSS whether in IMT normal group or IMT thickening group was approximately as follows: carotid sinus WSS $<$ WSS of CCA $<$ WSS of ICA, and WSS of anterior-lateral wall of carotid sinus $<$ WSS of posterior-interior wall. The internal distribution was stable. Therefore, it provides a useful tool for early and accurate prediction of the site of atherosclerosis in clinical.

Authors' contributions

Performed the literature review: YQM, BZ; Carried out echocardiography measurements: DF; Checked the validity of data: BZ, YQM; Data analysis: YQM, DF; Supported the experiments financially: BZ; Writied and revised the manuscript: BZ, YQM, DF. All authors read and approved the final manuscript.

\section{Acknowledgements}

Not applicable.

Competing interests

The authors declared that they have no competing interests.

Availability of data and materials

The datasets used during the current study are available from the corresponding author on reasonable request.

Consent for publication

Not applicable.

Ethics approval and consent to participate

The Shanghai East Hospital, China, gave the ethics approval for this study.

\section{Funding}

This study was funded by Pudong New Area Committee of Science and Technology (Grant No. PKJ2015-Y17), Important Weak Subject Construction Project of Pudong Health and Family Planning Commission of Shanghai (Grant No. PWZbr2017-09), and the National Natural Science Foundation of China (Grant No. 81571693).

\section{Publisher's Note}

Springer Nature remains neutral with regard to jurisdictional claims in published maps and institutional affiliations.

Received: 6 September 2018 Accepted: 10 October 2018

Published online: 19 October 2018

\section{References}

1. Li Q, Guo J, Cao XQ, Yuan X, Rao KQ, Zheng Z, Liu ZD, Hu SS. Trend of non-communicable disease mortality for three common conditions in the elderly population from 2002 to 2010: a-based study in China. Chronic Dis Transl Med. 2015;1(3):152-7.

2. Wang H, Qu M, Yang P, Yang B, Deng F. Dietary patterns and cardio-cerebrovascular disease in a Chinese population. Nutr Res Pract. 2015;9(3):313-8.

3. Hou Q, Li S, Gao Y, Tian H. Relations of lipid parameters, other variables with carotid intima-media thickness and plaque in the general Chinese adults: an observational study. Lipids Health Dis. 2018;17(1):107-20.

4. Cacciapaglia F, Zardi EM, Coppolino G, Buzzulini F, Margiotta D, Arcarese L, et al. Stiffness parameters, intima-media thickness and early atherosclerosis in systemic lupus erythematosus patients. Lupus. 2009;18(3):249-56.

5. Sarkar RN, Bhattacharya R, Bhattacharyya K, Paul R, Mullick OS. Adult onset Still's disease with persistent skin lesions complicated by secondary hemophagocytic lymphohistiocytosis. Int J Rheum Dis. 2014;17(1):118-21.

6. Millon A, Canet-Soulas E, Boussel L, Fayad Z, Douek P. Animal models of atherosclerosis and magnetic resonance imaging for monitoring plaque progression. Vascular. 2014;22(3):221-37. 
7. Diomedi M, Scacciatelli D, Misaggi G, Balestrini S, Balucani C, Sallustio F, et al. Increased common carotid artery wall thickness is associated with rapid progression of asymptomatic carotid stenosis. J Neuroimaging. 2015;24(5):473-8.

8. Villwock MR, Padalino DJ, Deshaies EM. Carotid artery stenosis with acute ischemic stroke: stenting versus angioplasty. J Vasc Interv Neurol. 2015;8(4):11-6.

9. Nezu T, Hosomi N, Aoki S, Matsumoto M. Carotid intima-media thickness for atherosclerosis. J Atheroscler Thromb. 2016;23(1):18-31.

10. Corti R, Fuster V. Imaging of atherosclerosis: magnetic resonance imaging. Eur Heart J. 2011;32(14):1709-819.

11. Veronique P, Sherwin SJ, Weinberg PD. Does low and oscillatory wall shear stress correlate spatially with early atherosclerosis? A systematic review. Cardiovasc Res. 2013;99(2):242-50

12. Nigro P, Abe Jl, Berk BC. Flow shear stress and atherosclerosis: a matter of site specificity. Antioxid Redox Signal. 2011:15(5):1405.

13. Slager CJ, Wentzel JJ, Gijsen FJ, Schuurbiers JC, Ac VDW, Af VDS, et al. The role of shear stress in the generation of rupture-prone vulnerable plaques. Nat Clin Pract Cardiovasc Med. 2005;2(8):401-7.

14. Hibi K, Honda Y, Kimura K, Umemura S. Atherosclerosis: progress in diagnosis and treatments. Topics: III. Nihon Naika Gakkai Zasshi. 2013;102(2):344-53.

15. Sunder GS, Benoit P, Arie B. Global stability analysis of flow through a fusiform aneurysm: steady flows. J Fluid Mech. 2014;752:90-106.

16. Owolabi MO, Agunloye AM, Ogunniyi A. The relationship of flow velocities to vessel diameters differs between extracranial carotid and vertebral arteries of stroke patients. J Clin Ultrasound. 2013;42(1):16-23.

17. De DG, Setacci F, Sirignano P, Galzerano G, Cappelli A, Setacci C. Optical coherence tomography after carotid stenting: rate of stent malapposition, plaque prolapse and fibrous cap rupture according to stent design. Eur J Vasc Endovasc Surg. 2013:45(6):579-87.

18. Wang C, Chen M, Liu SL, Liu Y, Jin JM, Zhang YH. Spatial distribution of wall shear stress in common carotid artery by color Doppler flow imaging. J Digit Imaging. 2013;26(3):466-71.

19. Fernández-Friera L, Ibáñez B, Fuster V. Imaging subclinical atherosclerosis: is it ready for prime time? A review. J Cardiovasc Transl Res. 2014;7(7):623-34.

20. Yang XL, Chen JC, Li JX, Cao J, Lu XF, Liu FC, et al. Risk stratification of atherosclerotic cardiovascular disease in Chinese adults. Chronic Dis Transl Med. 2016;2(2):102-9.

21. Cahill PA, Redmond EM. Vascular endothelium — gatekeeper of vessel health. Atherosclerosis. 2016;248:97-109.

22. Zhang B, Gu J, Qian M, Niu L, Zhou H, Ghista D. Correlation between quantitative analysis of wall shear stress and intima-media thickness in atherosclerosis development in carotid arteries. Biomed Eng Online. 2017;16(1):137-54

23. Blake JR, Meagher S, Fraser KH, Easson WJ, Hoskins PR. A method to estimate wall shear rate with a clinical ultrasound scanner. Ultrasound Med Biol. 2008:34(5):760-74.

24. Zhang F, Lanning C, Mazzaro L, Barker AJ, Gates PE, Strain WD, et al. In vitro and preliminary in vivo validation of echo particle image velocimetry in carotid vascular imaging. Ultrasound Med Biol. 2011:37(3):450-64.

25. Oshinski JN, Curtin JL, Loth F. Mean-average wall shear stress measurements in the common carotid artery. J Cardiovasc Magn Reson. 2006;8(5):717-22.

26. Bo Z, Sun Y, Xia L, Gu J. Time-dependent flow velocity measurement using two-dimensional color Doppler flow imaging and evaluation by Hagen-Poiseuille equation. Australas Phys Eng Sci Med. 2015;38(4):755-66.

27. Goddi A, Bortolotto C, Fiorina I, Raciti MV, Fanizza M, Turpini E, et al. High-frame rate vector flow imaging of the carotid bifurcation. Insights Imaging. 2017;8(3):319-28.

28. Papathanasopoulou P, Zhao S, Köhler U, Robertson MB, Long Q, Hoskins P, et al. MRI measurement of time-resolved wall shear stress vectors in a carotid bifurcation model, and comparison with CFD predictions. J Magn Reson Imaging. 2003;17(2):153-62.

29. Ooij PV, Potters WV, Collins J, Carr M, Carr J, Malaisrie SC, et al. Characterization of abnormal wall shear stress using 4D flow MRI in human bicuspid aortopathy. Ann Biomed Eng. 2015:43(6):1-13.

30. Spanos K, Petrocheilou G, Karathanos C, Labropoulos N, Mikhailidis D, Giannoukas A. Carotid bifurcation geometry and atherosclerosis. Angiology. 2017;68(9):757-64.

Ready to submit your research? Choose BMC and benefit from:

- fast, convenient online submission

- thorough peer review by experienced researchers in your field

- rapid publication on acceptance

- support for research data, including large and complex data types

- gold Open Access which fosters wider collaboration and increased citations

- maximum visibility for your research: over 100M website views per year

At BMC, research is always in progress.

Learn more biomedcentral.com/submissions 